\title{
Optimization of Indirect Fracturing Process Parameters Based on Mechanical Properties of Fractured and Low-Permeability Coal
}

\author{
Wenfeng Yuan, ${ }^{1,2}$ Xigui Zheng $\mathbb{D}^{1,3,4,5}$ and Niaz Muhammad Shahani $\mathbb{D}^{1,3}$ \\ ${ }^{1}$ School of Mines, China University of Mining and Technology, Xuzhou 221116, China \\ ${ }^{2}$ Changzhi Company of Shanxi Coal Transportation and Marketing Group, Changzhi 046000, China \\ ${ }^{3}$ The State Key Laboratory for Geo Mechanics and Deep Underground Engineering, China University of Mining \& Technology, \\ Xuzhou 221116, China \\ ${ }^{4}$ School of Mines and Civil Engineering, Liupanshui Normal University, Liupanshui, China \\ ${ }^{5}$ Guizhou Guineng Investment Co., Ltd., Guiyang, China
}

Correspondence should be addressed to Xigui Zheng; cumt_ckzxg@126.com

Received 12 August 2021; Revised 4 December 2021; Accepted 31 December 2021; Published 7 March 2022

Academic Editor: Yingfang Zhou

Copyright $(92022$ Wenfeng Yuan et al. This is an open access article distributed under the Creative Commons Attribution License, which permits unrestricted use, distribution, and reproduction in any medium, provided the original work is properly cited.

The coal-bed methane (CBM) resources in soft and low-permeability coals are assumed to be as much as $15 \times 10^{12} \mathrm{~m}^{3}$ in China. Indirect fracturing technology can be an effective method to successfully extract methane within soft coals. The key to the success of this technique is to optimize the parameters, such as water injection flow rate and fracture initiation location, so that the hydraulic fracturing parameters enable the fractures to pass through the interface between coal and rock and propagate sufficiently into the coal. This paper focuses on solving the above problems by focusing on discontinuities and plastic characteristics of soft coals. Voronoi polyhedron was used to simulate the discontinuities of coal, and the constitutive relations of ductile fractureseepage and elastoplastic damage-seepage are, respectively, given to the discontinuities and coal matrix. A numerical model was established based on the above theory to simulate the effect of stress difference $\Delta \sigma$, coal-rock interface friction coefficient $f_{c, r}$, water injection flow rate $i_{w}$, and distance between the well and the interface $D_{\mathrm{op}}$ on indirect fracturing fractures. The results show that the HFs area in the coal is positively correlated with $\Delta \sigma, f_{c, r}$, and $i_{w}$, and it first increases and then decreases with the decrease of $D_{\mathrm{op}}$. The above results were applied in the Zhaozhuang mine of Qinshui Basin by optimizing $D_{\mathrm{op}}=1 \mathrm{~m}$ and $i_{\mathrm{w}}=8 \mathrm{~m}^{3} / \mathrm{min}$, so that CBM production has been greatly increased. The results can provide theoretical support for the efficient development of CBM in fractured and low-permeability coal seam areas.

\section{Introduction}

The resources of fractured low-permeability coal account for $82 \%$ of the total coal in China, and the resources of coal-bed methane $(\mathrm{CBM})$ in coal is as high as $15 \times 10^{12} \mathrm{~m}^{3}[1,2]$. The efficient exploitation of this huge reserve of clean energy will help China achieve the peak of carbon dioxide emissions in 2030 and carbon neutrality in 2060 [3]. However, the fractured coal is generally of low mechanical parameters, abundant discontinuities, and low permeability because of small crack opening, leading to the failure of traditional fracturing into coal seams, shown as short hydraulic fracture (HF) extension and borehole collapse [4, 5]. Indirect fracturing technology can effectively avoid the disadvantages caused by the above direct fracturing coal technology. The basic step is to arrange the horizontal well in the hard roof and carry out perforation and hydraulic fracturing.

The key to the success of the technology is to optimize the indirect fracturing parameters so that HFs can be fully propagated into the coal seam. Numerical simulation is an important method to achieve the above objectives, however, its accuracy is restricted by two aspects: (1) constitutive equation and (2) coal discontinuities network model. For the first problem, the theory of linear elastic fracture mechanics was adopted by Zhang and Dontsov [6]. They found that the small stress difference and small elastic modulus difference 
of adjacent rock formation would lead to the interface strongly hindering the HF propagation. Based on the theory of elastic damage mechanics, Poludasu et al. [7] and Xue et al. [8] established a two-dimensional numerical model for the problem of HFs crossing the interface of the layered rock mass. They found that only when the interface strength is relatively high would HFs will pass through the interface. According to the CT test and the nonlinear mechanicsleakage hypothesis, Li et al. [9] developed a three-dimensional discontinuous network model and a plastic-nonlinear fracture-leakage-coupled constitutive formula. According to the numerical simulation results, Oyedere et al. [10] believe that in low-permeability media, too high a fracturing fluid injection rate will stop the propagation of hydraulic fractures. Based on the elastic damage mechanics, Guo et al. [11] studied the influence of multiple factors on the HF crossing the layered rock interface using the numerical simulation method. The results show that it is difficult for the HF to cross the interface under the conditions of low-stress difference and high material tensile strength.

For the second problem, scholars tend to simplify the fractured medium as an equivalent continuous medium (ECM) model. However, coal is naturally fractured [12]. Therefore, Vahab et al. [13] assumed that coal is a dual continuous medium. On this basis, a conclusion is obtained using the calculation of the extended finite element method, i.e., HFs are easier to cross the hard medium into the soft medium. In addition, some scholars have established the discrete fracture network model (DFN) of fractured media, however, they did not deeply study its mechanical properties. For example, $\mathrm{Ma}$ et al. [14] simplified coal discontinuity as matchsticks, where each stick represented one coal matrix, and the space between the sticks was representative of the discontinuities. Zhao et al. [15] regarded coal as masonry structures. Karimpouli et al. [16] made statistics of the distance and distribution of coal discontinuities and developed a rectangular grid model.

The above literature shows that there are abundant discontinuities with high permeability, however, poor mechanical properties in coal and HFs are easy to propagate in an adjacent rock formation in the conditions of high-stress differences, water injection flow rates, high interfacial strength, and significant differences of rock elastic modulus. However, scholars generally ignore the influence of an important parameter on indirect fracturing engineering, i.e., the distance between well and coal-rock interface $\left(D_{\mathrm{op}}\right)$. In addition, the elasticity theory and the simplified continuum model cannot fully reflect the characteristics of fractured and low-permeability coal, including abundant discontinuities and obvious plastic fracture behavior. Obviously, the hydraulic fracturing parameters optimized by traditional theory and model are not reliable.

Focusing on the mechanical properties of fractured and low-permeability coal, this paper studied the plastic damageseepage features of coal matrix, ductile fracture-seepage features, and distribution law of coal discontinuities. On this basis, the fluid-solid coupling constitutive equation of coal and the geometric model for discontinuity were established. Using the numerical simulation method, the propagation mechanism of HFs under the influence of four factors was studied, including $D_{\mathrm{op}}$, water injection rate, stress difference, and shear strength of coal-rock interface. The optimized parameters obtained by numerical simulation were applied to a coal mine, which greatly increases the output of CBM.

\section{Geological Characteristics and Extraction Status of CBM in Zhaozhuang Mine}

The proved reserves of CBM in Qinshui Basin account for more than $70 \%$ of China's CBM resources [17]. By direct fracturing coal technology, the southern Qinshui Basin has achieved average daily gas production of $1,000 \mathrm{~m}^{3} / \mathrm{d}$ per well. However, this mature technology cannot be replicated in the middle and east of the Qinshui basin because of the special geological conditions of CBM. In this paper, the Zhaozhuang mine (Figure 1) in the east of the Qinshui Basin is studied as an example.

The basic geological conditions of the CBM reservoir (No. 3 coal) in the Zhaozhuang mine are as follows: the thickness is $5 \mathrm{~m}$, and the depth is $450 \mathrm{~m}$. The gas content of the coal is $10 \mathrm{~m}^{3} / t$, and the reservoir pressure is $3.6-6.1 \mathrm{MPa}$. Under the influence of long-term and large-scale geological tectonic movement, 33 folds were formed in the mining area, which leads to changes in the stress state of the reservoir and the strength of the coal-rock interface. At the same time, abundant discontinuities are formed in coal under the action of strong stress, resulting in the formation of fractured and low-permeability coal. Generally, the compressive strength of coal is lower than $15 \mathrm{MPa}$, and its permeability is lesser than $1 \mathrm{mD}$.

In the early engineering practice, 245 direct fracturing wells have been drilled in the Zhaozhuang mine. Only 47 wells produce gas, and the average CBM production is less than $300 \mathrm{~m}^{3} / \mathrm{d}$, far from reaching the target of $3000 \mathrm{~m}^{3} / \mathrm{d}$. The main reason for the failure of direct fracturing technology is that the Zhaozhuang coal has the characteristics of abundant discontinuities and obvious plastic failure so that wells are easy to collapse, and HFs are difficult to extend far away, resulting in low CBM production.

However, the indirect fracturing coal technology (Figure 2) can effectively overcome the above difficulties. This new technology is strongly supported by CBM geological conditions, including vertical stress, which is the first principal stress. The rock layers gradually soften from top to bottom, which makes it possible for HFs in the roof to propagate to coal. However, so far, only two of the five indirectly fractured horizontal wells in the Zhaozhuang mine have achieved the goal of $3000 \mathrm{~m}^{3} / \mathrm{d}$.

The failure of the indirect fracturing technology is because HFs do not cross the coal-rock interface. The fundamental reasons are as follows: (1) from the mechanical theory, for the layered brittle rock formation, although almost all of the hydraulic energy is used to transform into fracture surface energy (i.e., elastic energy) to promote HF propagation. The interface between the rock layers will still strongly hinder HF propagation. However, the above phenomenon will be more obvious for fractured low-permeability coal because the hydraulic energy will also be 


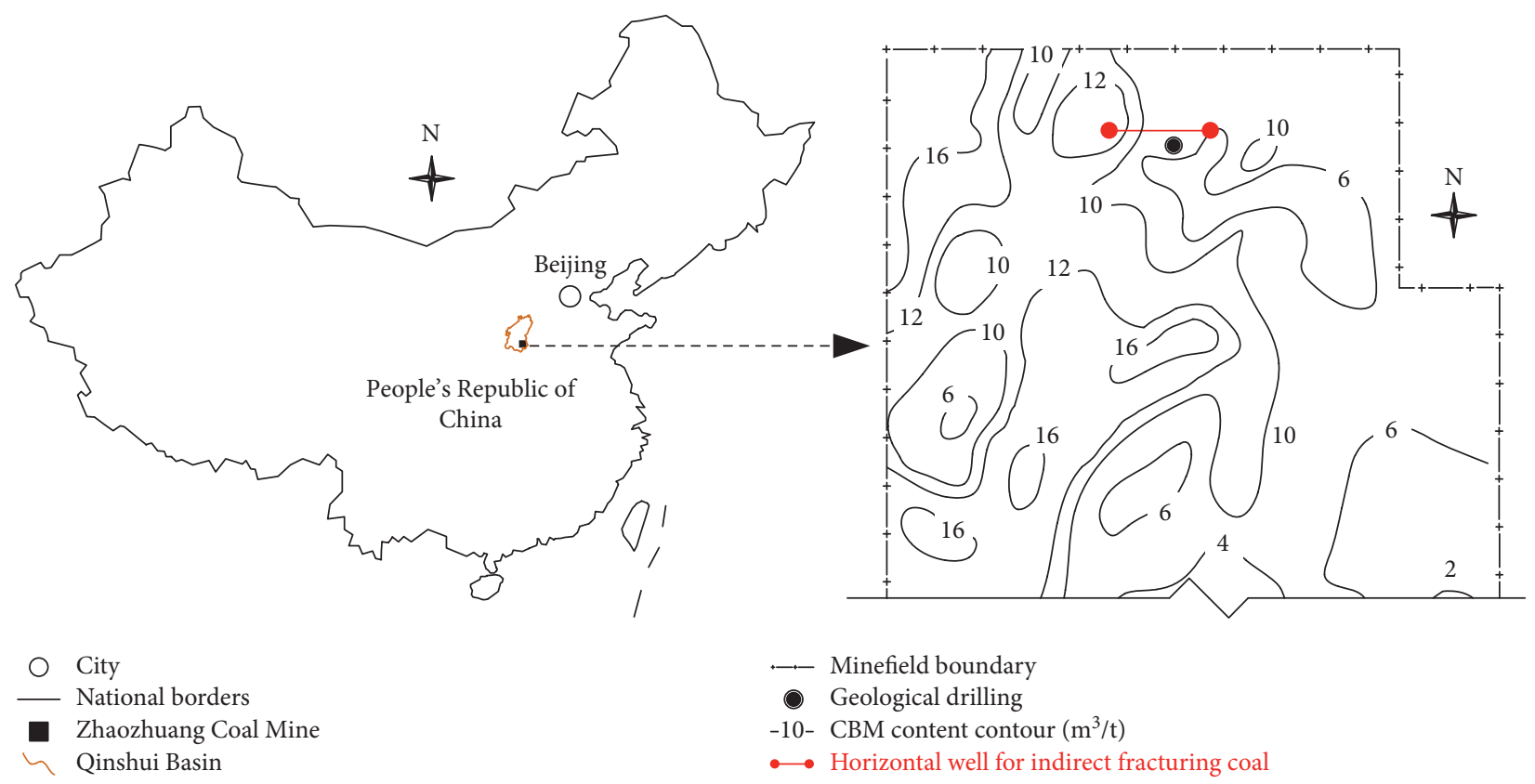

Figure 1: Location of Zhaozhuang mine and CBM occurrence environment.

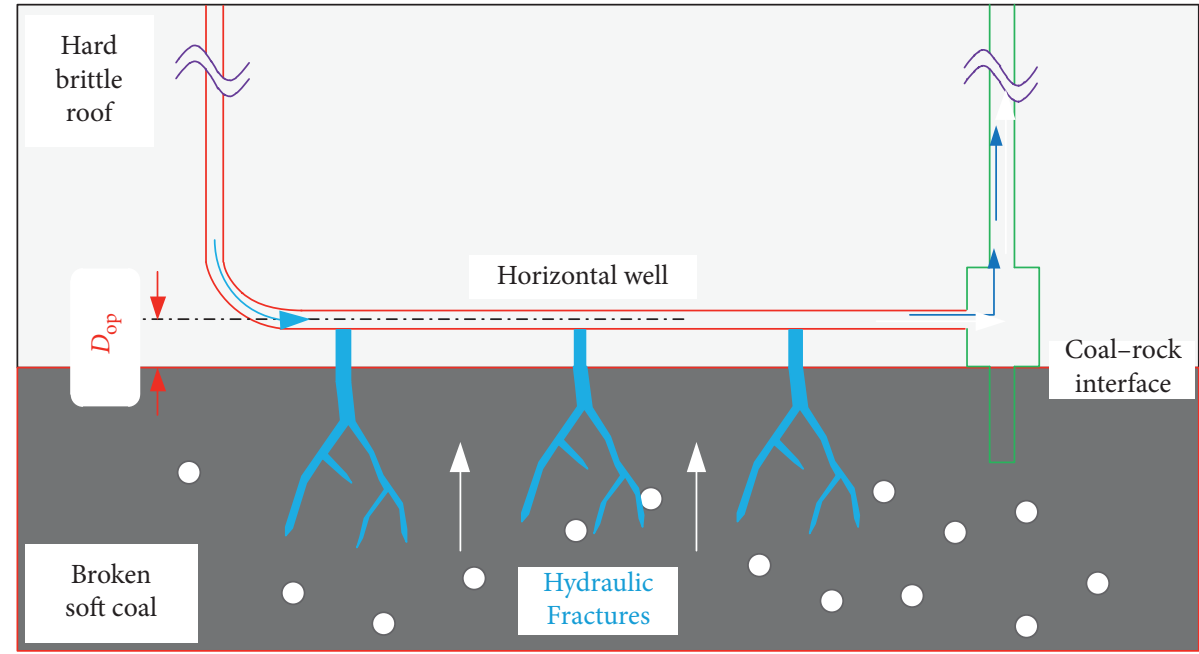

Figure 2: Schematic diagram of indirect fracturing coal technology.

consumed in the plastic work, which is not conducive to HF propagation [18]. Therefore, a new constitutive equation reflecting the toughness failure of coal needs to be established. (2) The coal discontinuities induce HFs to propagate along it [19], forming an energy-consuming mixed fracture mode. It is difficult for HFs to cross the coal-rock interface.

According to the above, the in-depth study of coal discontinuity and its fluid-solid coupling characteristics is the basis for further optimization of indirect fracturing technical parameters.

\section{The Fluid-Solid Coupling Theory Model of Pore-Fractured Coal}

Abundant discontinuous and significant nonlinear mechanical characteristics of plastic fracture-seepage are the two notable features of fractured low-permeability coal. The material is a typical pore (i.e., coal matrix)-fracture (i.e., coal discontinuities) medium [20]. There are three mechanical responses in the coal process during hydraulic fracturing [21], which are as follows: plastic damage-seepage of coal matrix, ductile fracture-seepage of coal discontinuities, and the stress-seepage interaction between them.

Considering that the mechanism of coal matrix failure is plastic deformation and crack propagation [22], the two factors should be included in the constitutive model. The specific steps are as follows:

The plastic damage constitutive relation of the coal matrix is as follows [19]:

$$
\sigma_{s}^{t}=(1-d): E_{0}:\left(\varepsilon_{s}-\varepsilon_{s}^{p}\right)+\alpha p_{w} I,
$$


where $\sigma_{s}^{t}$ is the total stress, $E_{0}$ is elastic stiffness matrix, $\varepsilon_{s}$ and $\varepsilon_{s}^{p}$ are total strain and plastic strain, $p_{w}$ is the pore water pressure, $I$ is the unit matrix, and $\alpha$ is the effective stress coefficient, and its expression is as follows:

$$
\alpha=1-\frac{K_{b}}{K_{s}}
$$

$K_{s}$ and $K_{b}$ are the effective bulk modulus of the solid constituent and drained bulk modulus of the porous medium, respectively.

The plastic strain in (2) is solved as follows:

The loading function is as follows:

$$
\begin{aligned}
F= & \frac{1}{1-A}\left(q_{\mathrm{eff}}-3 A p_{\mathrm{eff}}+B\left(\varepsilon_{s}^{p}\right) \cdot\left\langle\sigma_{\mathrm{eff}, i}^{\max }\right\rangle-C\left\langle-\sigma_{\mathrm{eff}, i}^{\max }\right\rangle\right) \\
& -\widehat{\sigma}_{\mathrm{eff}}\left(\varepsilon_{s}^{p}\right)=0,
\end{aligned}
$$

where $\quad A=\left(\sigma_{b} / \sigma_{u}-1\right) /\left(2 \sigma_{b} / \sigma_{u}-1\right), \quad B=\widehat{\sigma}_{\text {eff }}\left(\widehat{\varepsilon}_{s}^{p}\right) / \widehat{\sigma}_{\text {eff }}$ $(d) \cdot(1-A)-(1+A), \quad C=3\left(1-K_{c}\right) /\left(2 K_{c}-1\right), \quad p_{\text {eff }}=$ $\operatorname{trace}\left(\sigma_{\text {eff }}\right) / 3, \quad q_{\text {eff }}=\sqrt{2\left(S_{\text {eff }}: S_{\text {eff }}\right) / 3}, \quad S_{\text {eff }}=\sigma-p_{\text {eff }} I, \quad \sigma_{\text {eff }, i}^{\max }$ $(i=1,2,3)$ is the maximum effective principal stress, $\sigma_{b} / \sigma_{u}$ is the ratio of biaxial strength to uniaxial strength, and $\widehat{\sigma}_{\text {eff }}$ is the equivalent stress, and when $K_{c}=0.667$, the shape of loading function on the $\pi$ plane is close to Mohr-Coulomb yield criterion, as shown in Figure 3.

The plastic potential function is as follows:

$$
G=\sqrt{\left(\delta \sigma_{\mathrm{UTS}} \tan \psi\right)^{2}+q_{\mathrm{eff}}^{2}}-p_{\mathrm{eff}} \tan \psi
$$

where $\delta$ is a parameter, and $\delta=0.1$ in numerical simulation. $\sigma_{\text {UTS }}$ is the tensile strength, and $\psi$ is the dilatancy angle.

Based on the above equations, $\varepsilon_{s}^{\mathrm{p}}$ can be solved by the backward Euler method [23].

The damage evolution law of $d^{(+)}$and $d^{(-)}$in (1) can be obtained by the experiments (Section 4).

The permeability coefficient of the coal matrix changes as the strain increases. By fitting the experimental data of full stress-strain seepage, the relationship between the permeability coefficient and strain can be obtained. The experimental procedure refers to the literature [24]. The experimental results are shown in Figure 4. As the axial strain increases, the evolution of the permeability coefficient can be divided into three stages: (1) linear seepage, (2) rapid increase, and (3) nonlinear and stable stage of seepage stage.

By fitting the data in Figure 4, the expression of the evolution law of the permeability coefficient during the elastoplastic damage of the coal matrix can be obtained as follows:

$$
k=\left\{\begin{array}{l}
k_{\min }+k_{\min } \mathrm{e}^{\left[\left(\varepsilon_{\min }-\varepsilon\right) / \varepsilon_{\min }\right]^{\eta_{1}}} \text { (The first stage), } \\
k_{\min }+\delta_{1} k_{\min }\left[\left(\varepsilon-\varepsilon_{\min }\right) / \varepsilon_{\min }\right]^{\eta_{2}} \mathrm{e}^{\left[-\left(\varepsilon-\varepsilon_{\min }\right) / \varepsilon_{\min }\right]^{\eta_{3}}} \text { (The second stage), } \\
k_{\max } \mathrm{e}^{\left[-\left(\varepsilon_{\max }-\varepsilon\right) / \varepsilon_{\max }\right]^{\eta_{4}}}+\delta_{1} k_{\text {max }} \text { (The third stage). }
\end{array}\right.
$$

For coal discontinuities, this paper established the ductile fracture and seepage-coupled constitutive model under different fracture modes and assigned this attribute to cohesive elements. The steps for establishing the constitutive equations are as follows.

The constitutive equation at the stage of elastic deformation is [25]

$$
\sigma_{c}=D_{0, c} \varepsilon_{c}
$$

Once the following stress conditions are reached, cracks begin to form.

$$
\max \left\{\frac{\left\langle\sigma_{c, n}\right\rangle}{\sigma_{c, n}^{\text {peak }}}, \frac{\sigma_{c, s}}{\sigma_{c, s}^{\text {peak }}}, \frac{\sigma_{c, t}}{\sigma_{c, t}^{\text {peak }}}\right\}=1
$$

where $\sigma_{c, n}, \sigma_{c, s}$, and $\sigma_{c, t}\left(\right.$ or $\sigma_{c, n}^{\text {peak }}, \sigma_{c, s}^{\text {peak }}$, and $\left.\sigma_{c, t}^{\text {peak }}\right)$ are (peak) normal and tangential stress. The symbol is the Macaulay bracket. $D_{0, c}$ is the elastic stiffness matrix. $\varepsilon_{\mathrm{c}}$ is the strain vector. The relationship between $\varepsilon_{c}$ and separation vector $S$ is $\varepsilon_{c}=S / T_{0}$, and $T_{0}$ is the constitutive thickness of the cohesive element.

After the peak load, the constitutive relationship can be deduced by the following method.

Use Park-Paulino-Roesler (PPR) potential energy function [26] to derive stress-displacement response during the ductile fracture. The expression of the potential energy function $\Psi\left(S_{\mathrm{n}}, S_{\mathrm{S}}\right)$ is as follows:

$$
\Psi\left(S_{n}, S_{S}\right)=\left[\Gamma_{n}\left(1-\frac{S_{n}}{s_{n}}\right)^{\beta}\left(\frac{m}{\beta}+\frac{S_{n}}{s_{n}}\right)^{m}+\left\langle G_{n}-G_{S}\right\rangle\right] \times\left[\Gamma_{S}\left(1-\frac{\left|S_{S}\right|}{s_{S}}\right)^{\gamma}\left(\frac{n}{\gamma}+\frac{\left|S_{S}\right|}{s_{S}}\right)^{n}+\left\langle G_{S}-G_{n}\right\rangle\right]+\min \left(G_{n}, G_{S}\right)
$$

where $S_{\mathrm{S}}$ is the vector sum of $S_{s}$ and $S_{t}$, and $\Gamma_{\mathrm{n}}, \Gamma_{\mathrm{S}}$ are fracture energy constants. 


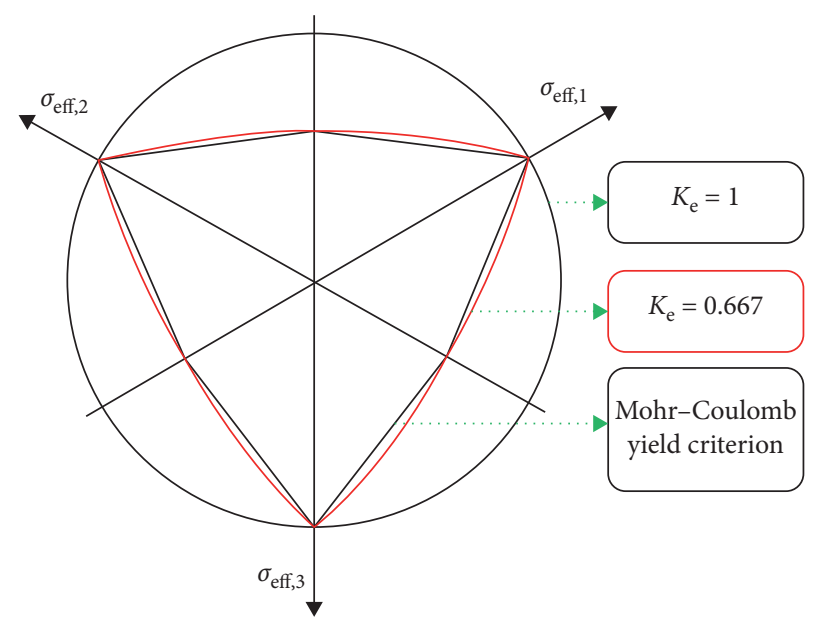

FIgURE 3: Yield curve.

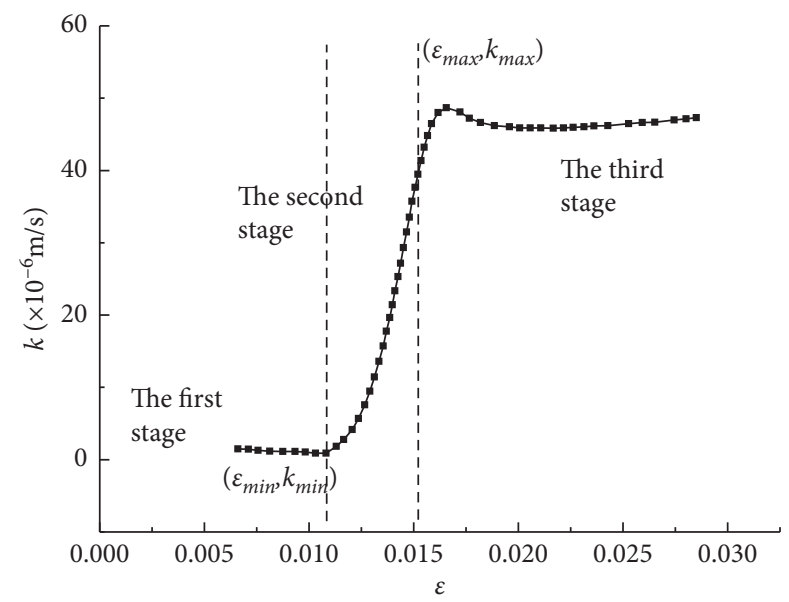

FIgURE 4: Evolution law of coal matrix permeability coefficient during the full stress-strain process.

$$
\left\{\begin{array}{l}
\Gamma_{n}=\left(-G_{n}\right)^{\left\langle G_{n}-G_{S}\right\rangle /\left(G_{n}-G_{S}\right)}(\beta / m)^{m}, \\
\Gamma_{S}=\left(-G_{S}\right)^{\left\langle G_{S}-G_{n}\right\rangle /\left(G_{S}-G_{n}\right)}(\gamma / n)^{n},
\end{array}\right.
$$

where $G_{n}$ and $G_{\mathrm{S}}$ are normal and tangential fracture energies, and $\beta$ and $\gamma$ are material parameters, which are obtained by fitting the traction force-separation curve. The parameters $m$ and $n$ are related to $\beta$ and $\gamma$. The expression of $m$ and $n$ is as follows:

$$
\left\{\begin{array}{l}
m=\beta(\beta-1) \chi_{\mathrm{n}}^{2} /\left(1-\beta \chi_{\mathrm{n}}^{2}\right), \\
n=\gamma(\gamma-1) \chi_{\mathrm{S}}^{2} /\left(1-\gamma \chi_{\mathrm{S}}^{2}\right),
\end{array}\right.
$$

where $\chi_{\mathrm{n}}$ and $\chi_{\mathrm{S}}$ represent the relative peak displacement. By calculating the first derivative of $\Psi\left(S_{\mathrm{n}}, S_{\mathrm{S}}\right)$, the constitutive equations of different fracture modes can be obtained, as shown in Eqs. 11 (13).

$$
\begin{aligned}
& \sigma_{c, n}=\frac{\Gamma_{n}}{s_{n}}\left\{m\left(1-\frac{S_{n}}{s_{n}}\right)^{\beta}\left(\frac{m}{\beta}+\frac{S_{n}}{s_{n}}\right)^{m-1}-\beta\left(1-\frac{S_{n}}{s_{n}}\right)^{\beta-1}\left(\frac{m}{\beta}+\frac{S_{n}}{s_{n}}\right)^{m}\right\} \times\left[\Gamma_{s}\left(1-\frac{S_{S}}{s_{S}}\right)^{\gamma}\left(\frac{n}{\gamma}+\frac{S_{S}}{s_{S}}\right)^{n}+\left\langle G_{S}-G_{n}\right\rangle\right], \\
& \sigma_{c, s}=\frac{\Gamma_{s}}{s_{S}}\left[\Gamma_{n}\left(1-\frac{S_{n}}{s_{n}}\right)^{\beta}\left(\frac{m}{\beta}+\frac{S_{n}}{s_{n}}\right)^{m}+\left\langle G_{n}-G_{s}-G_{t}\right\rangle\right] \times\left\{n\left(1-\frac{S_{S}}{s_{S}}\right)^{\gamma}\left(\frac{n}{\gamma}+\frac{S_{S}}{s_{S}}\right)^{n-1} \frac{S_{s}}{\sqrt{S_{s}^{2}+S_{t}^{2}}}-\gamma\left(1-\frac{S_{S}}{s_{S}}\right)^{\gamma-1}\left(\frac{n}{\gamma}+\frac{S_{S}}{s_{S}}\right)^{n} \frac{S_{s}}{S_{S}}\right\},
\end{aligned}
$$




$$
\sigma_{c, t}=\frac{\Gamma_{t}}{s_{S}}\left[\Gamma_{n}\left(1-\frac{S_{n}}{s_{n}}\right)^{\beta}\left(\frac{m}{\beta}+\frac{S_{n}}{s_{n}}\right)^{m}+\left\langle G_{n}-G_{s}-G_{t}\right\rangle\right] \times\left\{n\left(1-\frac{S_{S}}{s_{S}}\right)^{\gamma}\left(\frac{n}{\gamma}+\frac{S_{S}}{s_{S}}\right)^{n-1} \frac{S_{t}}{\sqrt{S_{s}^{2}+S_{t}^{2}}}-\gamma\left(1-\frac{S_{S}}{s_{S}}\right)^{\gamma-1}\left(\frac{n}{\gamma}+\frac{S_{S}}{s_{S}}\right)^{n} \frac{S_{t}}{S_{S}}\right\}
$$

Thus, the constitutive equation of the ductile fracture under the mixed fracture mode is obtained, and pure mode I and pure mode II fractures can be regarded as special cases of them.

According to the stress data obtained from the above constitutive model, the elastic energy $G^{\mathrm{e}}$ and inelastic energy $G^{\text {in }}$ fracture energy can be obtained by integrating the displacement.

$$
\begin{aligned}
G^{e} & =\frac{\left(\sigma_{c, n}^{0} s_{n}^{e}+\sigma_{c, s}^{0} s_{n}^{e}+\sigma_{c, t}^{0} s_{n}^{e}\right)}{2}, \\
G^{\text {in }} & =G_{n}+G_{s}+G_{t}-G^{e, p e a k},
\end{aligned}
$$

where $\sigma_{c, n}^{0}\left(s_{n}^{e}\right)$ is the stress (separation) in elastic deformation stage, respectively. $G^{\text {epeak }}$ is the maximum elastic energy.

Coal discontinuities are the main seepage channels of fluid [27]. In addition to the mechanical equation of coal discontinuities, the tangential and normal seepage equations of water in discontinuities should also be given. Considering that fluid migration in fractures often exhibits nonlinear characteristics, the tangential flow equation is established based on the Forchheimer equation.

$$
-\nabla p_{t}=\frac{12 \mu}{l w^{3}} Q_{t}+\beta_{w} \frac{\rho}{(l w)^{2}} Q_{t}^{2}
$$

At the same time, the fluid seepage along the normal direction of the fracture cannot be ignored, especially after the coal matrix is damaged. The expression is as follows:

$$
Q_{n}=2 k\left(p_{n, \text { cen }}-p_{n, \text { boun }}\right),
$$

where $-\nabla p_{\mathrm{t}}$ is the pressure gradient, $\mu$ is the dynamic viscosity, $w$ is the gap width, $Q_{\mathrm{t}}$ is total flow, $\beta_{\mathrm{w}}=3.35 \times$ $10^{-15} \mathrm{~kg} / \mathrm{s}^{2}$ is the non-Darcy flow factor, $\rho_{\mathrm{w}}$ is water density, $Q_{\mathrm{n}}$ is the leak-off flow, $k$ is the permeability coefficient (Eq. $5)$, and $p_{\mathrm{n} \text {,cen }}$ and $p_{\mathrm{n} \text {,boun }}$ are the water pressure in the middle and boundary gap, respectively.

Based on above equations, DF-S constitutive equations are established, and the numerical calculation process is shown in Figure 5.

\section{Parameter Identification and Constitutive Model Verification}

4.1. Experimental Results of Fracture Mechanics. The fracture mechanics experiment shown in Figure 6 [28] is an important method to obtain coal fracture parameters and verify the rationality of the constitutive equation. In addition, the mechanical parameters of the coal matrix are obtained using loading and unloading experiments and seepage experiments [29]. The results are shown in Table 1 and Figure 7. Establish a numerical calculation model consistent with the fracture experiment and the cohesive elements arranged along the fracture surface, and its mechanical properties are controlled by equations described in Sec. 3. The comparison result of numerical simulation and experiment is shown in Figure 6.

Using the above comparison, we find that the PF-S constitutive equations can better describe the mechanical properties of fractured coal.

4.2. Hydraulic Fracturing Experiment and Simulation. Zhaozhuang coal is rich in discontinuities, which is the main propagation channel of HFs. Therefore, before numerical simulation, it is necessary to establish a discrete fracture network model close to the actual discontinuities.

According to related research [30], we use the Voronoi polyhedron to simplify the spatial distribution of coal discontinuities. The geometric parameters of the Voronoi polyhedron are obtained by CT scanning (Figure 8(a)), including discontinuous spacing, and the respective fluctuation amplitudes. Thus, the reconstructed discontinuities spatial distribution is obtained (Figure 8(b)). Comparing figures $8(\mathrm{a})$ and $8(\mathrm{~b})$, it can be seen that the Voronoi polyhedron is similar to the discontinuities network geometry obtained from the CT experiment.

Based on the above geometric model, the constitutive equation can be further verified by hydraulic fracturing experiments. The boundary conditions of the hydraulic fracturing experiment are shown in Table 2, and the test results are shown in figures 9(a) and 9(b) [21]. The numerical model is established according to figure $9(\mathrm{a})$, in which the discontinuities in cement are arranged in the middle and parallel to $\sigma_{\mathrm{H}}$, and the discontinuities in coal are consistent with figure $8(\mathrm{~b})$. The constitutive equations in Sec. 3 were applied to coal, and parameters are shown in Table 1 and Figure 6. The elastic modulus and strength of cement are 3 times that of coal, however, the fracture displacement is 0.5 times that of coal. The friction coefficient of the interface is taken according to the literature [21]. The excess pore water pressure was set to 0 , the pore ratio of cement and coal was 0.2 and 0.14 , and the saturation was 1 . The results of experiments and simulation are shown in Figure 9.

According to the above results, two conclusions can be summarized: (1) the stress difference threshold of hydraulic fracture passing through the coal-rock interface is $6 \mathrm{MPa}$. (2) The simulation results of the pressure-time curve (figures 9(c) and 9(e)), based on the third constitutive model, are close to the experimental results, indicating the rationality of the constitutive model. 


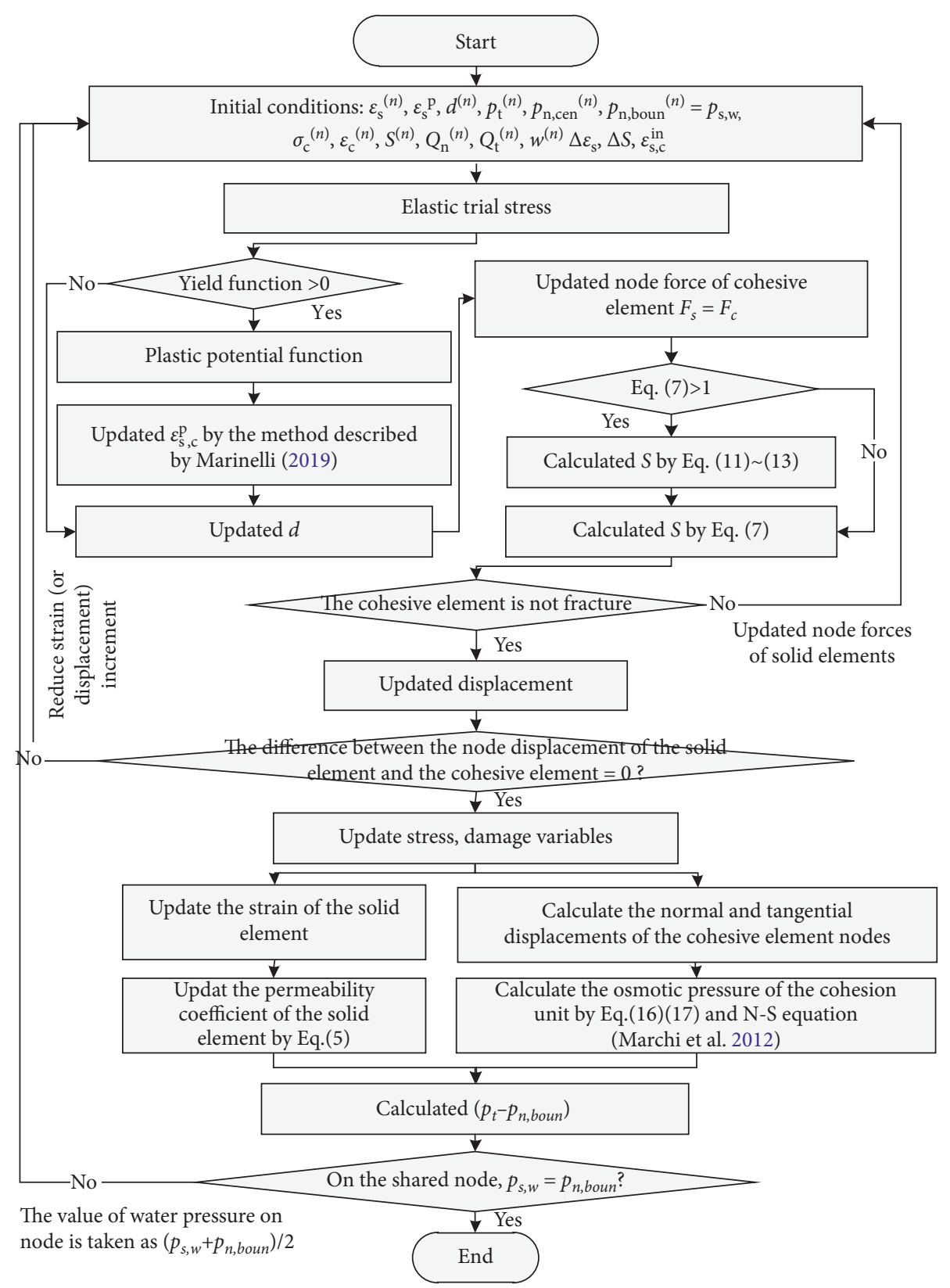

FIGURE 5: Numerical calculation process.

\section{Optimization of Indirect Fracturing Process Parameters}

Based on the numerical simulation in Section 4, we further study the influence of stress difference $\Delta \sigma$, coal-rock interface friction coefficient $f_{c, r}$, drilling and interface spacing $D_{\text {op }}$, and water flow $i_{w}$ parameters on the effect of indirect fracturing coal technology under the condition of field-scale.

The numerical models with length, width, and height of $20 \mathrm{~m}, 14 \mathrm{~m}$, and $10 \mathrm{~m}$ are established. Among them, the discontinuity in the rock is located in the middle of the model, and coal discontinuities are modeled according to the discrete fracture network in Sec. 3.3. As the geological structure of the Zhaozhuang Mine is relatively small, it has a little impact on the discontinuity distribution shown in
Figure 8. The water injection point in the model was located at the distance of $D_{\text {op }}=0.5 \mathrm{~m}$ to $2.5 \mathrm{~m}$ from the coal-rock interface, and the water injection flow was $8 \mathrm{~m}^{3} / \mathrm{min}$. The stress difference $\left(\Delta \sigma=\sigma_{\mathrm{v}}-\sigma_{\mathrm{h}}\right)$ was set as 4,6 , and $8 \mathrm{MPa}$ by changing the interface friction coefficient until the HF passes through the interface. The numerical results are shown in Figure 10.

The numerical simulation reflects two results: (1) the stress difference $\Delta \sigma$ and interface friction coefficient $f_{\mathrm{c}, \mathrm{r}}$ are the key factors to determine whether HFs can cross the coalrock interface. Taking $D_{\text {op }}=0.5 \mathrm{~m}$ and $i_{w}=8 \mathrm{~m}^{3} / \mathrm{min}$ as an example, when $\Delta \sigma$ increases from $4 \mathrm{MPa}$ to $8 \mathrm{MPa}$, the critical friction coefficient $f_{c, r}$ decreases from 0.22 to 0.02 . Under the conditions of other values of $\Delta \sigma$ and $D_{\text {op }}$, the critical $f_{c, r}$ also has similar changes. (2) $D_{\text {op }}, i_{\mathrm{w}}$ will have a 


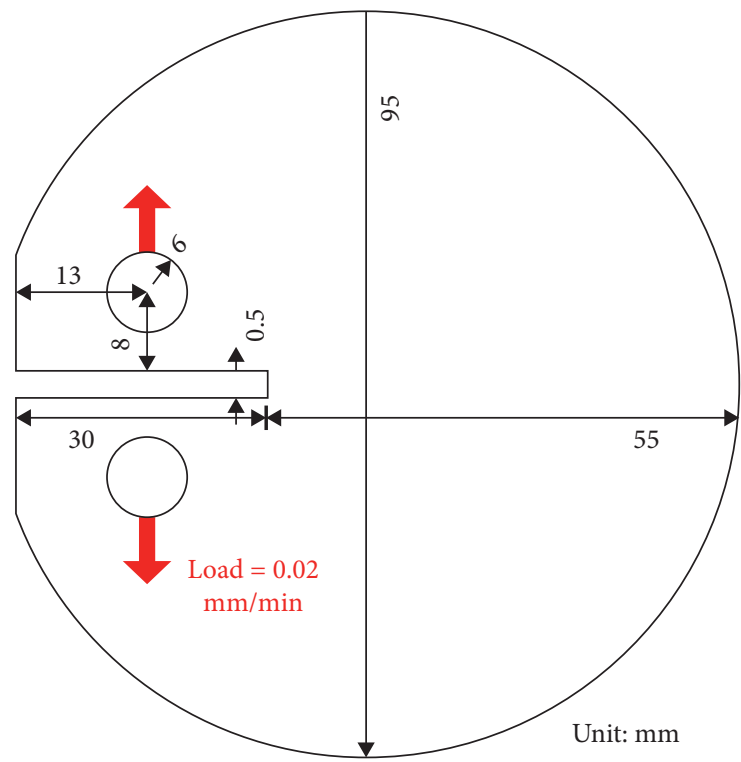

(A)

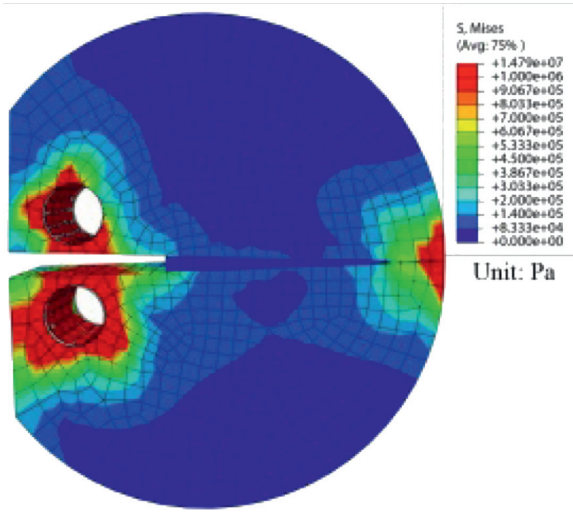

(C)

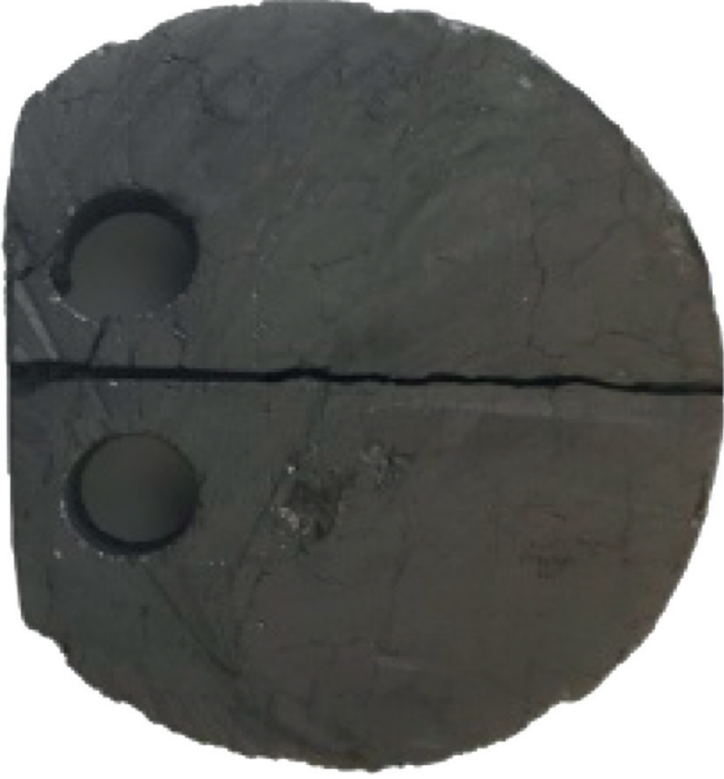

(B)

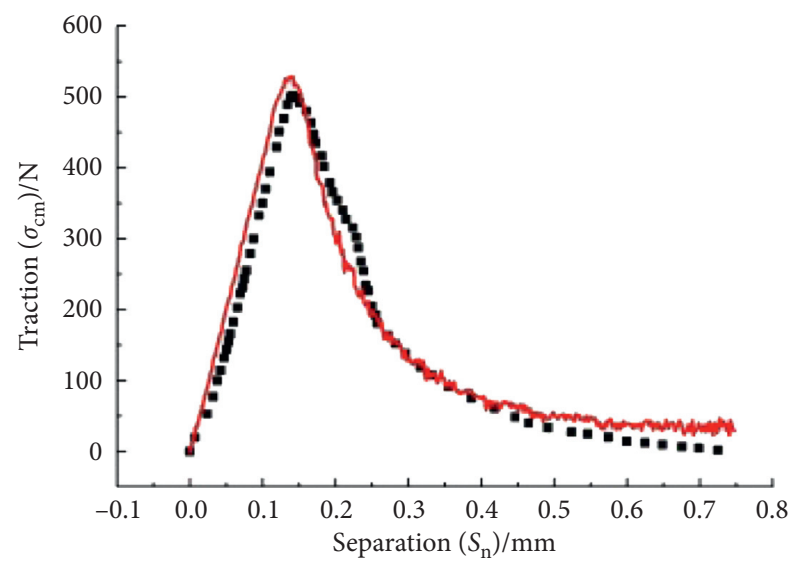

- Test data of mode I fracture

Numerical simulation data of mode I fracture

(D)

(a)

Figure 6: Continued. 


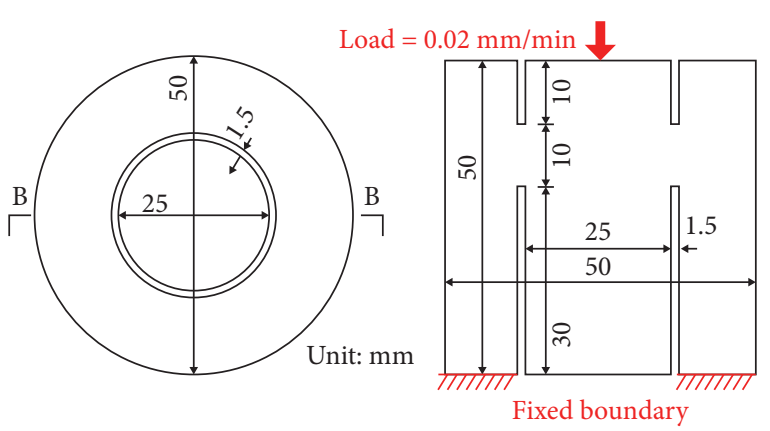

(A)

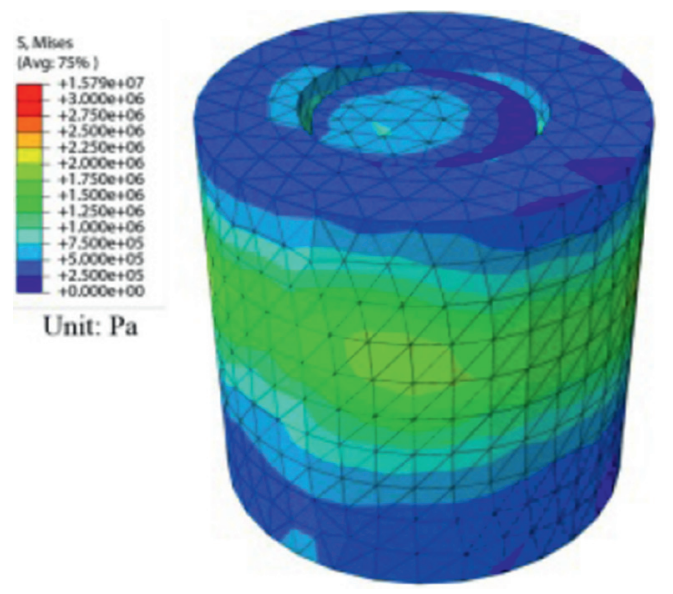

(C)

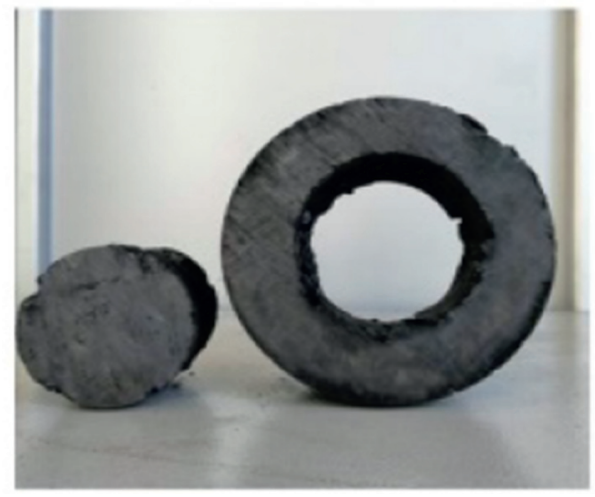

(B)

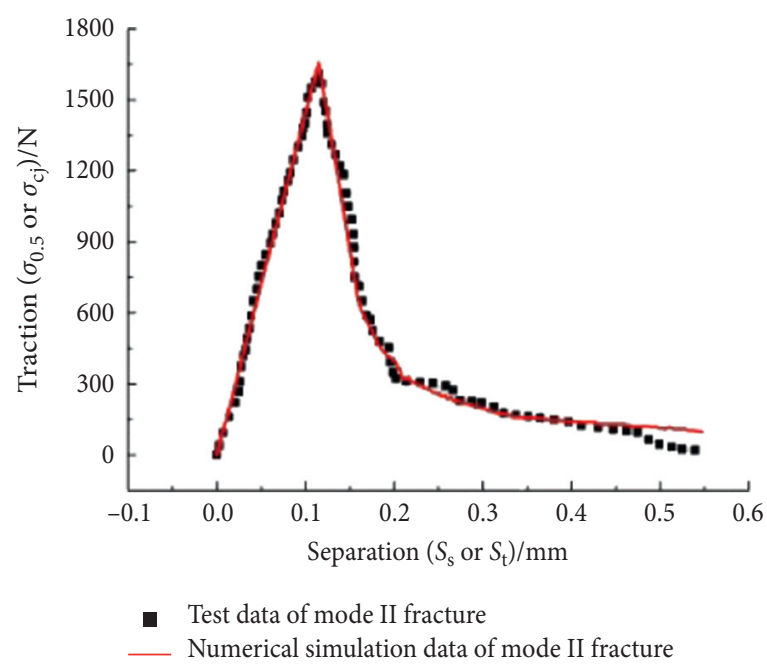

(D)

(b)

FiguRE 6: Numerical simulation and experimental results. (a) Numerical simulation and experimental results of mode I fracture. (A) Size of a specimen or numerical model with mode I fracture. (B) Specimen and fracture pattern of test [2]. (C) Fracture pattern from numerical calculation. (D) Comparison of traction-separation curves obtained by two methods. (b) Numerical simulation and experimental results of mode II fracture. (A) Size of a specimen or numerical model with mode II fracture. (The left one is the top view, and the right one is the B-B section. (B) Specimen and fracture pattern of laboratory test [2]. (C) Numerical calculation model and fracture pattern. (D) Comparison of traction-separation curves obtained by two methods.

significant impact on the HF area in coal. Taking $i_{\mathrm{w}}=8 \mathrm{~m}^{3} /$ min and $\Delta \sigma=6 \mathrm{MPa}$ as an example, the HF area decreases rapidly with the increase of $D_{\text {op }}$ from $1 \mathrm{~m}$ to $2.5 \mathrm{~m}$. When $D_{\text {op }}$ decreases from $1 \mathrm{~m}$ to $0.5 \mathrm{~m}$, a single $\mathrm{HF}$ form is gradually formed by the hydraulic fracture network. In other words, under the condition of numerical simulation, $D_{\text {op }}=1 \mathrm{~m}$ will maximize the hydraulic fracture area. Furthermore, the HF area will increase with the increase of $i_{\mathrm{w}}$. Take $D_{\text {op }}=1 \mathrm{~m}$ and $\Delta \sigma=6 \mathrm{MPa}$ as an example. With the increase of $i_{\mathrm{w}}$ from $4 \mathrm{~m}^{3} / \mathrm{min}$ to $12 \mathrm{~m}^{3} / \mathrm{min}$, a complex fracture network is gradually formed in the coal, and the HF area increases rapidly.
Summarizing the above phenomena, it can be found that the HF area in coal is positively correlated with $i_{w}, f_{c, r}, \Delta \sigma$, and the law of first-increasing-and-then-decreasing with the increase of $D_{\mathrm{op}}$-increasing.

In terms of engineering practice (Figure 11), the stress field in the coal mine area and the mechanical strength of coal-rock interface are judged according to the geological drilling data, and the areas with large $\Delta \sigma$ and $f_{c, r}$ were selected. On this basis, the value of $D_{\mathrm{op}}=1 \mathrm{~m}$ and $i_{\mathrm{w}}=8 \mathrm{~m}^{3} /$ min was taken, and then drainage and gas production were carried out. In situ experiments show that after the above indirect fracturing process optimization, the daily output of 
TABle 1: Mechanical parameters.

\begin{tabular}{|c|c|c|c|c|c|c|c|c|c|c|c|c|c|c|c|}
\hline & $\phi(\%)$ & $\begin{array}{c}\mathrm{E}_{0} \\
(\mathrm{GPa})\end{array}$ & $\mu$ & $\eta_{1}$ & $\eta_{2}$ & $\eta_{3}$ & $\begin{array}{l}\Psi \\
\left({ }^{\circ}\right)\end{array}$ & $\sigma_{\mathrm{b} 0} / \sigma_{\mathrm{c} 0}$ & $\mathrm{~K}_{\mathrm{c}}$ & $\sigma_{\mathrm{UTS}}$ & $\delta$ & $\varepsilon_{\min }$ & $\varepsilon_{\max }$ & $\underset{\left(10^{-6} \mathrm{~ms}^{-1}\right)}{\mathrm{k}_{\min }}$ & $\underset{\left(10^{-6} \mathrm{~ms}^{-1}\right)}{\mathrm{k}_{\max }}$ \\
\hline Coal matrix & 8.9 & 1.61 & 0.3 & -0.4 & 8.6 & 0.5 & 20 & 2.0 & 0.667 & 0.1 & 0.1 & 0.012 & 0.016 & 0.03 & 52 \\
\hline \multirow[t]{2}{*}{ Roof } & 7.9 & 6.58 & 0.2 & -0.1 & 10 & 0.1 & 28 & 1.5 & 0.667 & 4.70 & 0.1 & 0.008 & 0.01 & 0.01 & 87 \\
\hline & $\begin{array}{c}E_{n} \\
(\mathrm{GPa})\end{array}$ & $\begin{array}{c}\mathrm{E}_{\mathrm{ss}} \\
(\mathrm{GPa})\end{array}$ & $\begin{array}{c}\mathrm{E}_{\mathrm{tt}} \\
(\mathrm{GPa})\end{array}$ & $\begin{array}{c}\sigma_{c, n}^{0} \\
(\mathrm{MPa})\end{array}$ & $\begin{array}{c}\sigma_{c, s}^{0} \\
(\mathrm{MPa})\end{array}$ & $\begin{array}{c}\sigma_{c, t}^{0} \\
(\mathrm{MPa})\end{array}$ & T0 & $\begin{array}{c}\mathrm{s}_{\mathrm{n}} \\
\left(10^{-4} \mathrm{~m}\right)\end{array}$ & $\begin{array}{c}\mathrm{s}_{\mathrm{S}} \\
\left(10^{-4} \mathrm{~m}\right)\end{array}$ & $\mathrm{s}_{\mathrm{n}, \mathrm{p}}\left(10^{-4} \mathrm{~m}\right)$ & $\mathrm{s}_{\mathrm{S}, \mathrm{p}}\left(10^{-4} \mathrm{~m}\right)$ & & & & \\
\hline \multirow[t]{2}{*}{$\begin{array}{l}\text { Coal } \\
\text { discontinuity }\end{array}$} & 0.98 & 1.83 & 1.83 & 0.67 & 1.06 & 1.06 & 1 & 3.2 & 5.9 & 0.61 & 1.78 & & & & \\
\hline & B & $\gamma$ & $\chi_{\mathrm{n}}$ & $\chi_{\mathrm{s}}$ & $\begin{array}{c}\mathrm{G}_{\mathrm{n}} \\
(\mathrm{N} / \mathrm{m})\end{array}$ & $\begin{array}{c}\mathrm{GS} \\
(\mathrm{N} / \mathrm{m})\end{array}$ & $\mathrm{m}$ & $\mathrm{n}$ & $\Gamma_{\mathrm{n}}$ & $\Gamma_{\mathrm{S}}$ & $\begin{array}{c}1 / \mathrm{m}^{3} \\
(\mathrm{~s} \cdot \mathrm{MPa})\end{array}$ & & & & \\
\hline $\begin{array}{l}\text { Coal } \\
\text { discontinuity }\end{array}$ & 3.56 & 3.36 & 0.19 & 0.29 & 57.74 & 343.05 & 0.3 & 0.94 & 2.72 & -1132.5 & $1.2 \times 10^{-10}$ & & & & \\
\hline
\end{tabular}

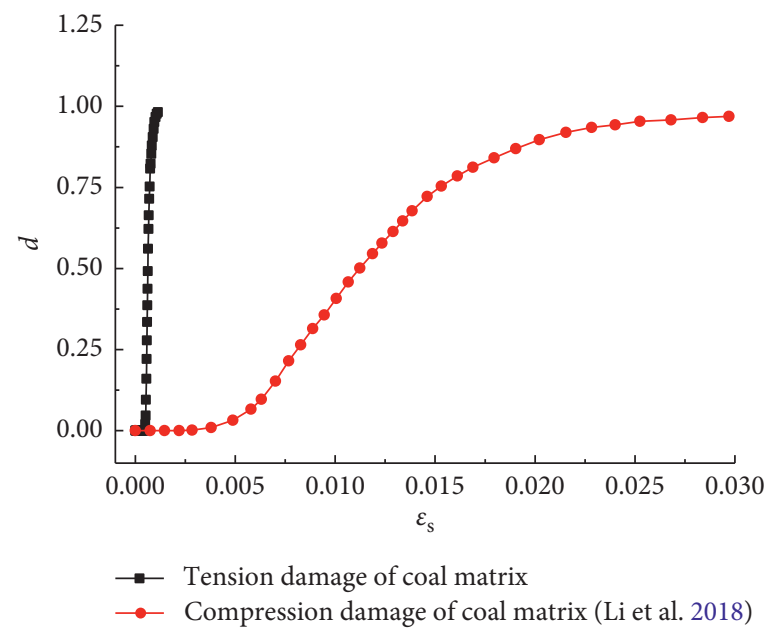

FIGURE 7: Damage mechanical properties coal matrix.

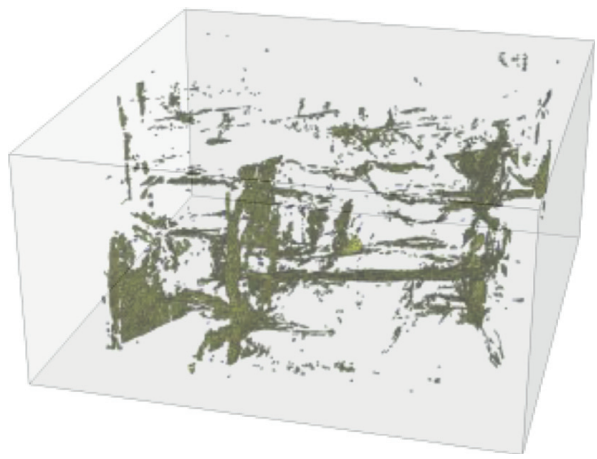

(a)

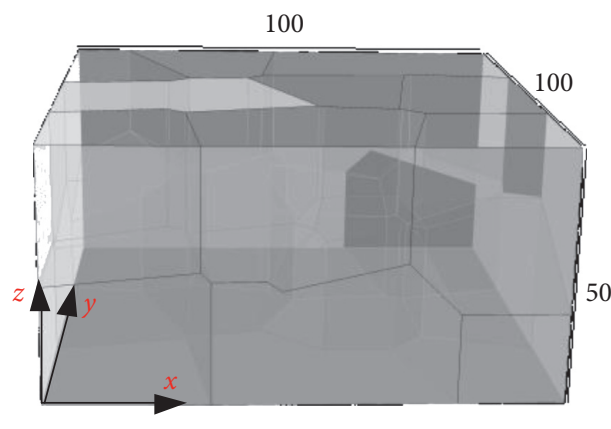

(b)

FIGURE 8: CT scanning and discontinuities network model of fractured coal. (a) Discontinuities in coal sample. (b) Reconstruction model of discontinuities.

CBM was increased from $1000 \mathrm{~m}^{3}$ to more than $5000 \mathrm{~m}^{3}$, as shown in Figure 12.

\section{Discussion}

In this paper, the plastic fracture-seepage constitutive relation and discrete fracture network model of the fractured coal were established, which were verified by fracture mechanics, hydraulic fracturing experiment, and CT experiment. On this basis, a calculation model was established. The evolution law of the HF area in the coal seam with stress difference $\Delta \sigma$, interface friction coefficient $f_{\mathrm{c}, \mathrm{r}}$, spacing between the well and interface $D_{\text {op }}$, and water injection flow $i_{\mathrm{w}}$ was studied. The simulation results show that higher $f_{\mathrm{c}, \mathrm{r}}$ and $\Delta \sigma$ values are helpful for HFs to cross the coal-rock interface, while larger $i_{\mathrm{w}}$ and appropriate $D_{\mathrm{op}}$ will help form a complex fracture network in the coal seam and improve $\mathrm{CBM}$ production. As far as we know, it is the first time to 
TABLE 2: Stress state of hydraulic fracturing experiments.

\begin{tabular}{lccc}
\hline Specimen number & & Stress $(\mathrm{MPa})$ & $\sigma_{\mathrm{H}}$ \\
& $\sigma_{\mathrm{v}}$ & 5 & 3 \\
$1 \#$ & 9 & 5 & 3 \\
$2 \#$ & 9 & 5 & 3 \\
$3 \#$ & 8 & 5 & 3 \\
$4 \#$ & 8 & 5 & 3 \\
$5 \#$ & 7 & 5 & \\
\hline
\end{tabular}

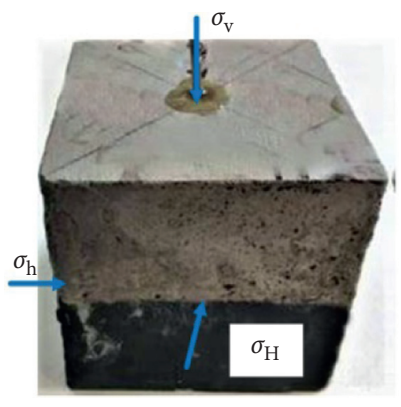

(a)

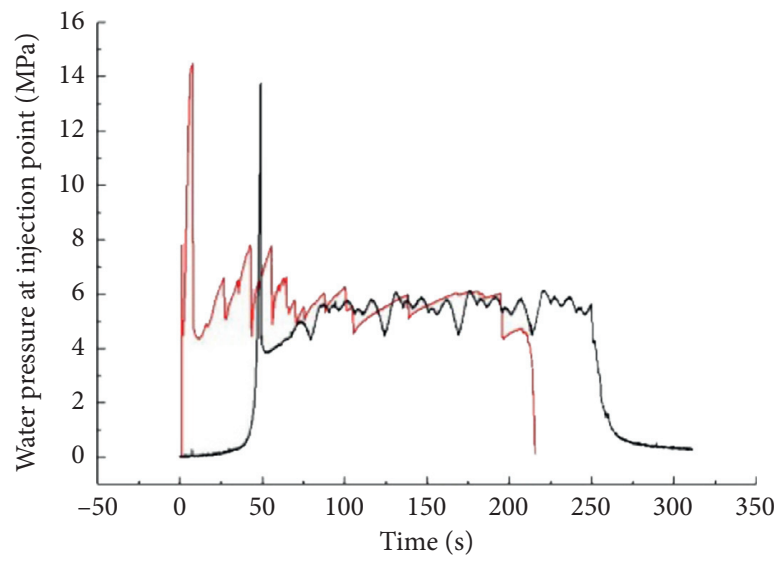

Experimental results Simulation results

(c)

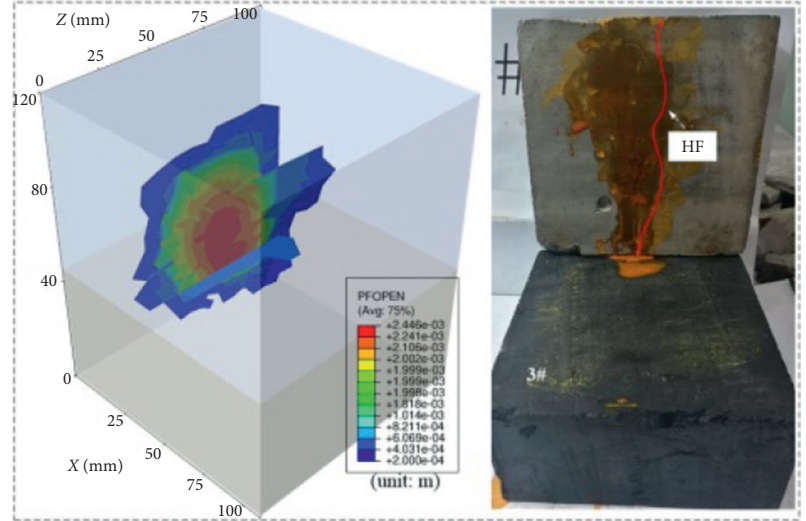

(b)

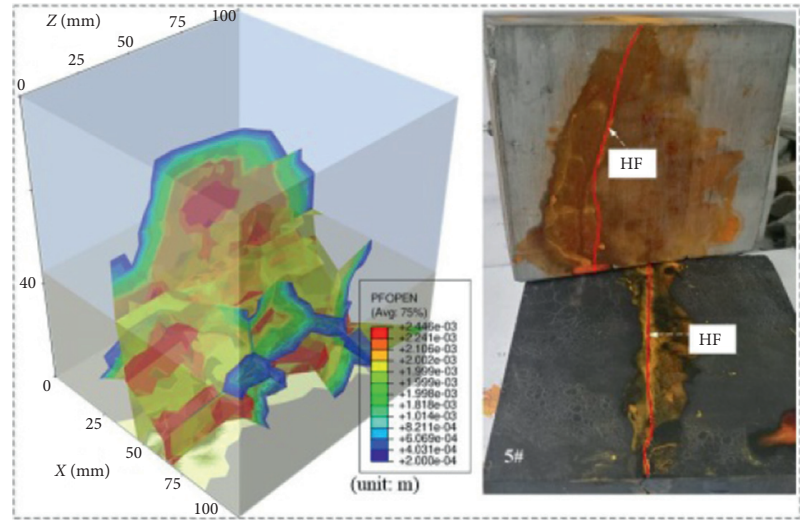

(d)

FIGURE 9: Continued. 


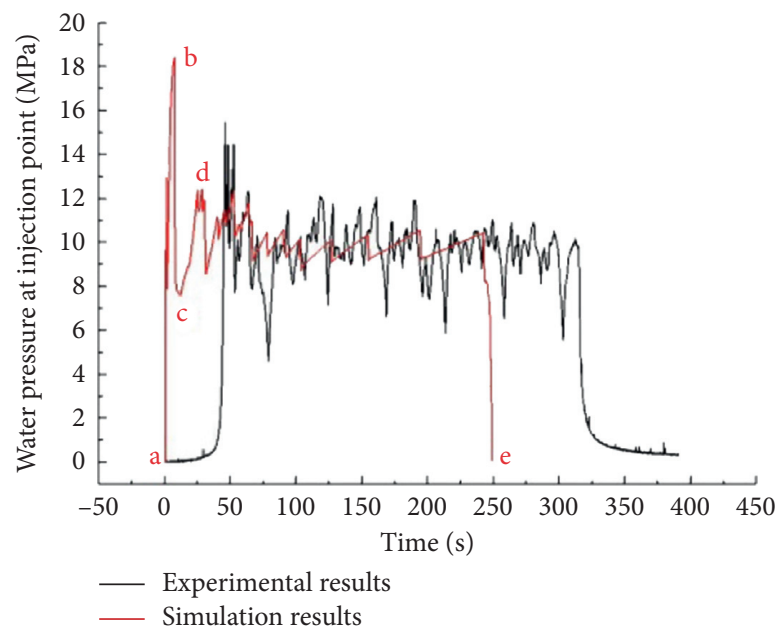

(e)

Figure 9: Results of experiments and simulation. (a) Specimen [21]. (b) Simulation results of HF opening displacement (left, unit: m) and fracture pattern of 3\# specimen (right, [21]) (stress state: $\sigma_{v}, \sigma_{H}, \sigma_{h}=6,5,3 \mathrm{MPa}$. (c) Water pressure-time curves at injection injection point obtained from results of simulation and experiments (3\# specimen). (d) Simulation results of HF opening displacement (left, unit: $\mathrm{m}$ ) and fracture pattern of $5 \#$ specimen (right, [21]) (Stress state: $\sigma_{v}, \sigma_{H}, \sigma_{h}=9,5,3 \mathrm{MPa}$ ). (e) Pressure-time curves at water injection point obtained from numerical simulation and HF experiments (1\# specimen).

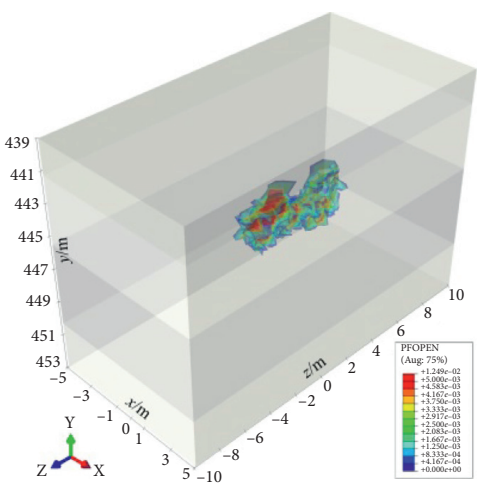

(a)

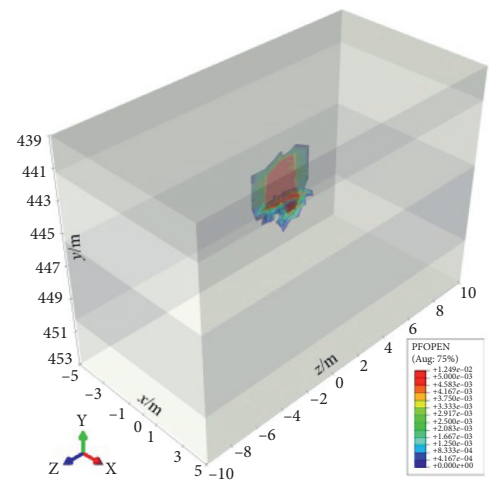

(d)

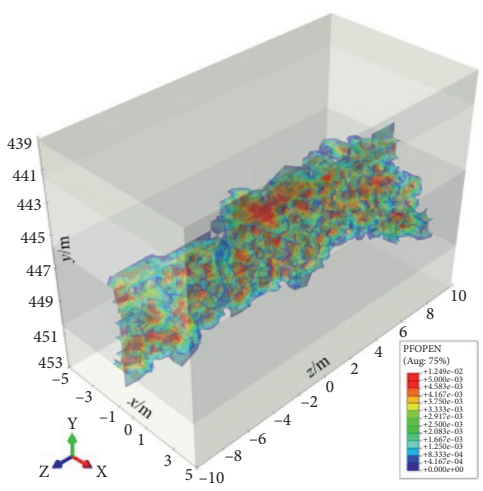

(b)

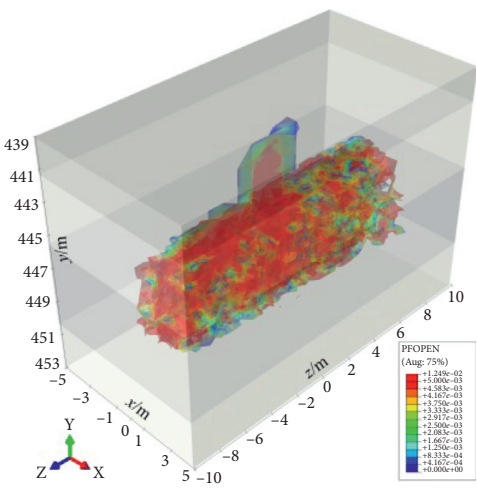

(e)

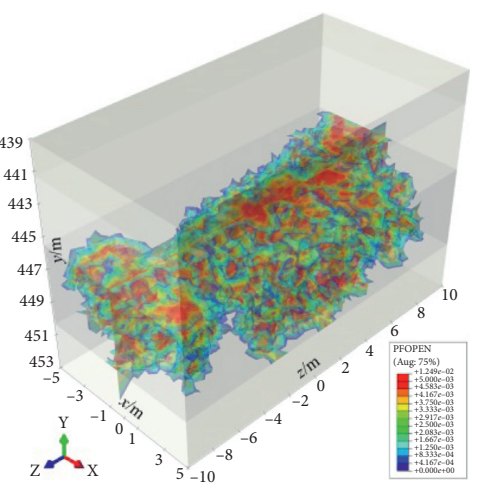

(c)

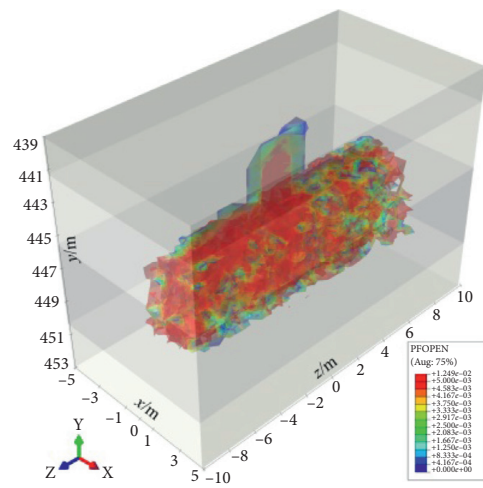

(f)

Figure 10: Continued. 


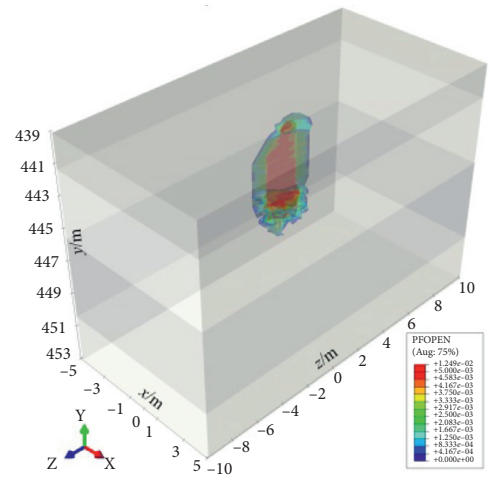

(g)

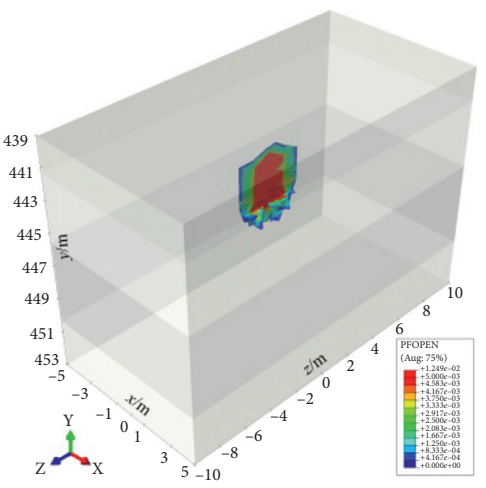

(j)

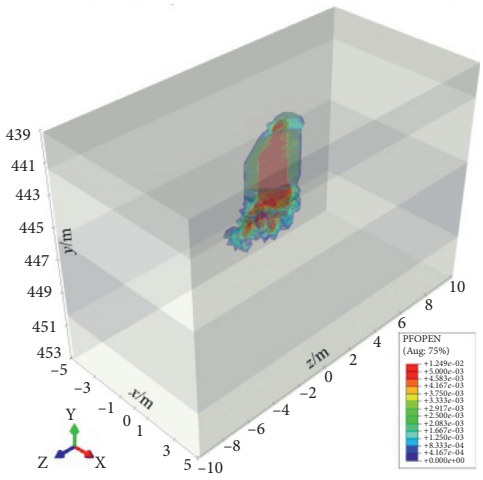

(h)

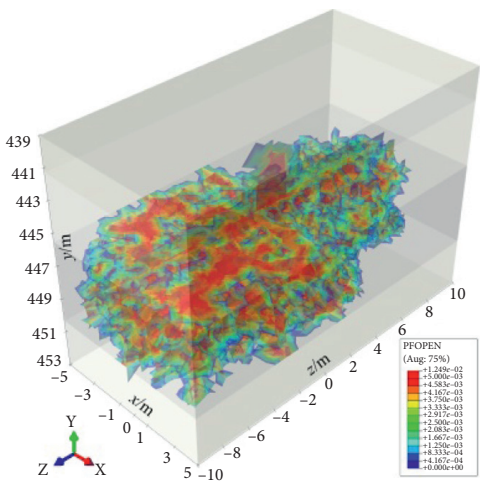

(k)

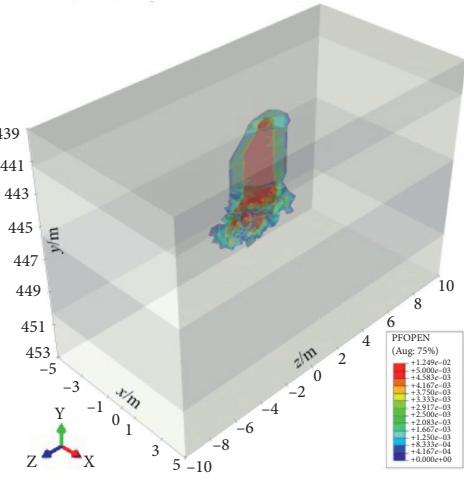

(i)

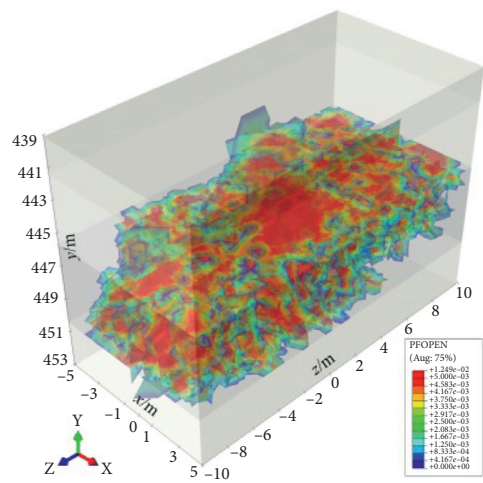

(1)

Figure 10: Numerical simulation results of indirect fracturing coal technology. (a) HFs opening $\left(D_{o p}=0.5 \mathrm{~m}, \Delta \sigma=4 \mathrm{MPa}, f_{c x}=0.22\right.$, $i_{w}=8 \mathrm{~m}^{3} / \mathrm{min}$. (b) HFs opening $\left(D_{o p}=0.5 \mathrm{~m}, \Delta \sigma=6 \mathrm{MPa}, f_{c, x}=0.05, i_{w}=8 \mathrm{~m}^{3} / \mathrm{min}\right)$. (c) HFs opening $\left(D_{o p}=0.5 \mathrm{~m}, \Delta \sigma=4 \mathrm{MPa}, f_{c x}=0.22\right.$, $i_{w}=8 \mathrm{~m}^{3} / \mathrm{min}$. (c) HFs opening $\left(D_{o p}=0.5 \mathrm{~m}, \Delta \sigma=8 \mathrm{MPa}, f_{c x}=0.22, i_{w}=8 \mathrm{~m}^{3} / \mathrm{min}\right.$. (d) HFs opening $\left(D_{o p}=0.5 \mathrm{~m}, \Delta \sigma=4 \mathrm{MPa}, f_{c x}=0.72\right.$, $i_{w}=8 \mathrm{~m}^{3} / \mathrm{min}$. (e) HFs opening $\left(D_{o p}=1.5 \mathrm{~m}, \Delta \sigma=6 \mathrm{MPa}, f_{c x}=0.53, i_{w}=8 \mathrm{~m}^{3} / \mathrm{min}\right.$. (f) HFs opening $\left(D_{o p}=1.5 \mathrm{~m}, \Delta \sigma=8 \mathrm{MPa}, f_{c x}=0.40\right.$, $i_{w}=8 \mathrm{~m}^{3} / \mathrm{min}$. (g) HFs opening $\left(D_{o p}=2.5 \mathrm{~m}, \Delta \sigma=4 \mathrm{MPa}, f_{c x}=0.90, i_{w}=8 \mathrm{~m}^{3} / \mathrm{min}\right.$. (h) HFs opening $\left(D_{o p}=2.5 \mathrm{~m}, \Delta \sigma=6 \mathrm{MPa}, f_{c x}=0.70\right.$, $i_{w}=8 \mathrm{~m}^{3} / \mathrm{min}$. (i) HFs opening $\left(D_{o p}=2.5 \mathrm{~m}, \Delta \sigma=8 \mathrm{MPa}, f_{c x}=0.71, i_{w}=8 \mathrm{~m}^{3} / \mathrm{min}\right.$. (j) HFs opening $\left(D_{o p}=1.0 \mathrm{~m}, \Delta \sigma=6 \mathrm{MPa}, f_{c x}=0.52\right.$, $i_{w}=4 \mathrm{~m}^{3} / \mathrm{min}$. (k) HFs opening $\left(D_{o p}=1.0 \mathrm{~m}, \Delta \sigma=6 \mathrm{MPa}, f_{c x}=0.14, i_{w}=8 \mathrm{~m}^{3} / \mathrm{min}\right.$. (l) HFs opening $\left(D_{o p}=1.0 \mathrm{~m}, \Delta \sigma=6 \mathrm{MPa}, f_{c x}=0.14\right.$, $i_{w}=12 \mathrm{~m}^{3} / \mathrm{min}$.

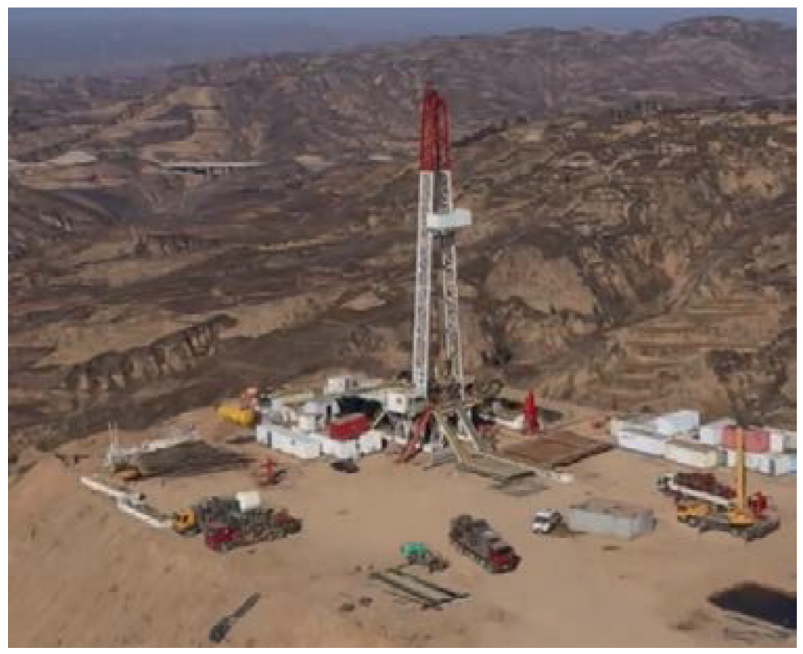

(a)

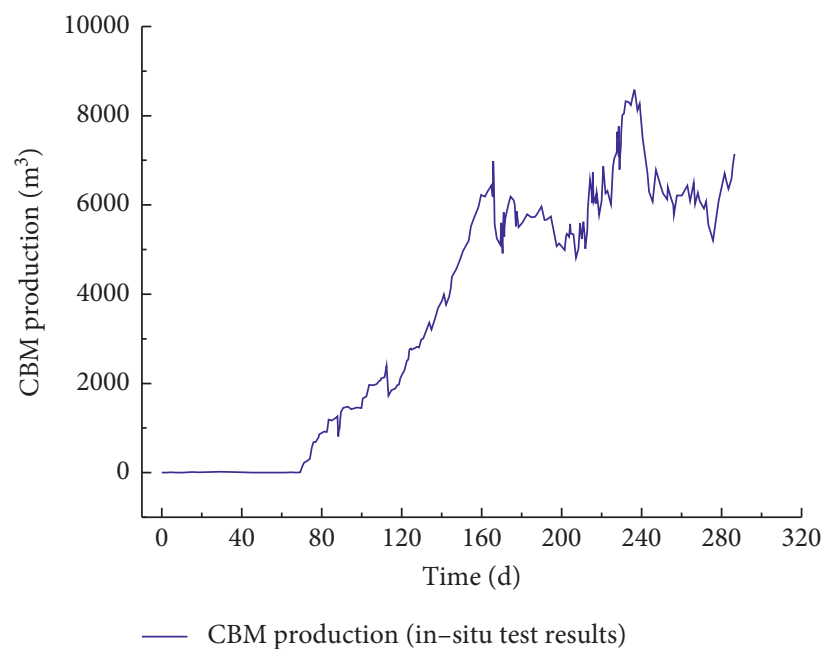

(b)

FIGURE 11: In situ test of indirect fracturing and CBM production. (a) Field test. (b) CBM production. 


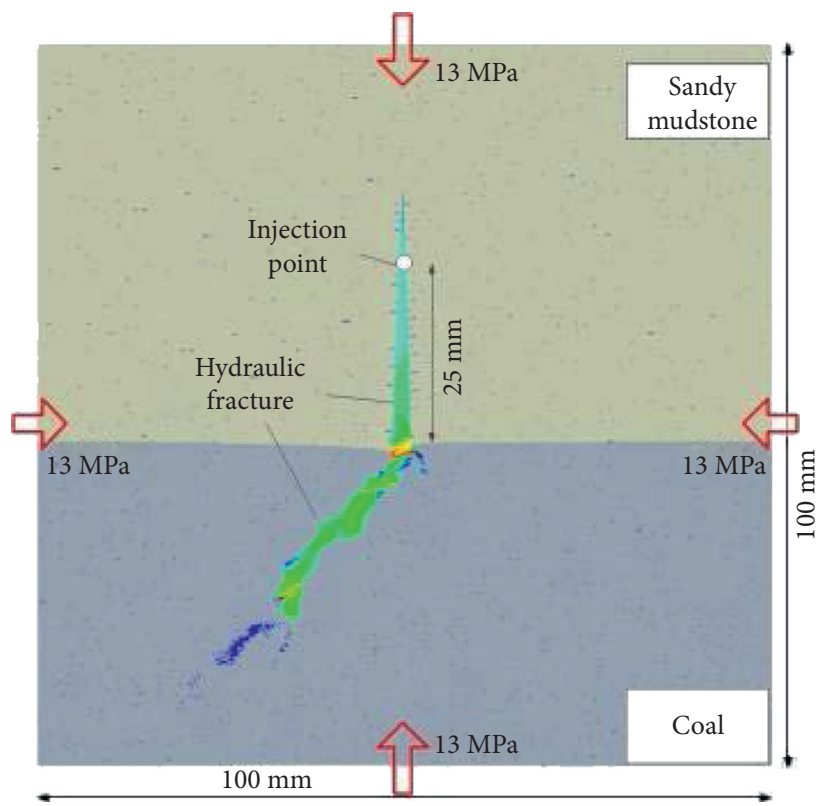

FIGURE 12: Hydraulic fracturing results obtained from 2D numerical model.

combine the plastic fracture-seepage constitutive relation and the discrete fracture network model of the coal discontinuities to carry out the three-dimensional numerical model and then optimize the parameters of indirect fracturing coal technology.

The fractured coal has the characteristics of plastic deformation and ductile fracture, which will cause complex nonlinear seepage response in the coal matrix and the coal discontinuities. Obviously, the theory of linear elastic fracture mechanics [7], elastic damage mechanics [11], and fluid-solid coupling model based on Darcy's law [31] cannot fully reflect the nonlinear mechanics and seepage characteristics mentioned above. At the same time, most numerical models do not consider the joint effect of $D_{\mathrm{op}}, i_{\mathrm{w}}$ on HF area in coal. The numerical calculation model based on the constitutive relation (Section 3) and considering the influence of $D_{\mathrm{op}}$ and $i_{\mathrm{w}}$ make the results more meaningful.

In addition to the constitutive equations, another issue worthy of attention is the $3 \mathrm{D}$ discrete fracture network model of coal. At present, the material was mostly simplified into an equivalent continuous medium model [32] and a dual-porous medium model [33], or a 2D discrete fracture network (DFN) model [16]. However, the results obtained by the $2 \mathrm{D}$ numerical model may mislead the optimization of indirect fracturing process parameters. When the geological parameters and fracturing process parameters of the $2 \mathrm{D}$ model and the 3D model are the same, the HFs in the roof terminate at the coal-rock interface. Only when the stress difference reaches $10 \mathrm{MPa}$, the HFs will propagate into the coal (Figure 12). Obviously, the results obtained from the $2 \mathrm{D}$ model are far from the results of the $3 \mathrm{D}$ model and the laboratory test.

Based on the constitutive relationship and discrete fracture network model in this paper, a numerical model of indirect fracturing coal technology was established, and the influence of stress difference, interface friction coefficient, spacing between the well and interface, and the water injection flow on the HF area was studied. The results show that $\Delta \sigma$ and $f_{\mathrm{c}, \mathrm{r}}$ are the key parameters to ensure that HFs cross the coal-rock interface, and the larger they are, the easier the HF is to enter the coal seam. More importantly, it is not that the smaller the $D_{\text {op }}$, the larger the HF area in the coal seam, however, there is an optimal $D_{\text {op }}$ value. By optimizing the above indirect fracturing parameters, the production of CBM is greatly improved.

\section{Conclusions}

Upon applying the optimized parameters of indirect fracturing coal technology, the CBM production was greatly increased. The results obtained in this paper could facilitate increased CBM production in fractured low-permeability coal regions and help China achieve its "carbon neutrality by $2060^{\prime \prime}$ goal. The main conclusions of this study can be summarized as follows:

(1) The nonlinear characteristics of fractured low-permeability coal, including plastic deformation, ductile fracture, and seepage, can be well-represented by PF$S$ constitutive relationship.

(2) The remarkable nonlinear failure characteristic of fractured coal is the root cause of hydraulic fractures that are difficult to cross the coal-rock interface.

(3) Increasing the water injection flow rate and reducing the spacing between the well and the coal-rock interface can greatly improve the success rate of indirect fracturing projects.

\section{Data Availability}

The data are available on request. 


\section{Conflicts of Interest}

The authors declare that there are no conflicts of interest regarding the publication of this paper.

\section{Acknowledgments}

This research was funded by 2017 Special Project of Subject Frontiers Scientific Research in China University of Mining and Technology (2017XKQY047).

\section{References}

[1] K. Dong, "Study on geological conditions affected to production of coalbed methane well of broken soft coal reservoir," Dissertation, CCTEG China Coal Research Institute, China, 2019.

[2] Y. Li, J. Yang, Z. Pan, S. Meng, K. Wang, and X. Niu, "Unconventional natural gas accumulations in stacked deposits: a discussion of upper paleozoic coal-bearing strata in the east margin of the ordos basin, China," Acta Geologica Sinica - English Edition, vol. 93, no. 1, pp. 111-129, 2019.

[3] Y. Xue, J. Liu, P. G. Ranjith, X. Liang, and S. Wang, "Investigation of the influence of gas fracturing on fracturing characteristics of coal mass and gas extraction efficiency based on a multi-physical field model," Journal of Petroleum Science and Engineering, vol. 206, Article ID 109018, 2021.

[4] Y. Li, J. Yang, Z. Pan, and W. Tong, "Nanoscale pore structure and mechanical property analysis of coal: an insight combining AFM and SEM images," Fuel, vol. 260, Article ID 116352, 2020.

[5] S. Tao, Z. Pan, S. Tang, and S. Chen, "Current status and geological conditions for the applicability of CBM drilling technologies in China: a review," International Journal of Coal Geology, vol. 202, pp. 95-108, 2019.

[6] F. Zhang and E. Dontsov, "Modeling hydraulic fracture propagation and proppant transport in a two-layer formation with stress drop," Engineering Fracture Mechanics, vol. 199, pp. 705-720, 2018.

[7] S. Poludasu, O. Awoleke, M. Ahmadi, and C. Hanks, "Using experimental design and response surface methodology to model induced fracture geometry in Shublik shale," Journal of Unconventional Oil and Gas Resources, vol. 15, pp. 43-55, 2016.

[8] Y. Xue, T. Teng, F. Dang, Z. Ma, S. Wang, and H. Xue, "Productivity analysis of fractured wells in reservoir of hydrogen and carbon based on dual-porosity medium model," International Journal of Hydrogen Energy, vol. 45, no. 39, pp. 20240-20249, 2020.

[9] H. Li, W. G. Liang, and Y. L. Jiang, "Numerical study on the field-scale criterion of hydraulic fracture crossing the interface between roof and broken low-permeability coal," Rock Mechanics and Rock Engineering, 2021.

[10] M. Oyedere, K. Gray, and M. W. McClure, "Numerical investigation of a novel hypothesis for fracture termination and crossing, with applications to lost circulation mitigation and hydraulic fracturing," Journal of Unconventional Oil and Gas Resources, vol. 15, pp. 122-133, 2016.

[11] J. Guo, B. Luo, C. Lu, J. Lai, and J. Ren, "Numerical investigation of hydraulic fracture propagation in a layered reservoir using the cohesive zone method," Engineering Fracture Mechanics, vol. 186, pp. 195-207, 2017.

[12] E. M. Llanos, R. G. Jeffrey, R. Hillis, and X. Zhang, "Hydraulic fracture propagation through an orthogonal discontinuity: a laboratory, analytical and numerical study," Rock Mechanics and Rock Engineering, vol. 50, no. 8, pp. 2101-2118, 2017.

[13] M. Vahab, S. Akhondzadeh, A. R. Khoei, and N. Khalili, "An $\mathrm{X}$-FEM investigation of hydro-fracture evolution in naturallylayered domains," Engineering Fracture Mechanics, vol. 191, pp. 187-204, 2018.

[14] Q. Ma, S. Harpalani, and S. Liu, "A simplified permeability model for coalbed methane reservoirs based on matchstick strain and constant volume theory," International Journal of Coal Geology, vol. 85, no. 1, pp. 43-48, 2011.

[15] J.-J. Zhao, Y. Zhang, and P. G. Ranjith, "Numerical modelling of blast-induced fractures in coal masses under high in-situ stresses," Engineering Fracture Mechanics, vol. 225, p. 106749, 2020.

[16] S. Karimpouli, P. Tahmasebi, H. L. Ramandi, P. Mostaghimi, and M. Saadatfar, "Stochastic modeling of coal fracture network by direct use of micro-computed tomography images," International Journal of Coal Geology, vol. 179, pp. 153-163, 2017.

[17] X. Z. Zhao, Y. H. Yang, and F. J. Sun, "Enrichment mechanism and exploration and development technologies of high rank coalbed methane in south Qinshui Basin, Shanxi Province," Petroleum Exploration and Development, vol. 43, no. 2, pp. 303-309, 2016.

[18] H. Li, W. Liang, and Y. Wu, "Study on the mechanism of coalrock interface hindering hydraulic fracture propagation in the process of indirectly fracturing broken soft coal seam," IOP Conference Series: Earth and Environmental Science, vol. 861, no. 6, Article ID 062104, 2021.

[19] H. Li, W. G. Liang, and G. F. Li, "The ductile failure-seepage coupling constitutive equations of broken soft coal and its and its verification in indirect fracturing engineering," Journal of China Coal Society, 2021.

[20] M. Cheng, X. Fu, and J. Kang, "Compressibility of different pore and fracture structures and its relationship with heterogeneity and minerals in low-rank coal reservoirs: an experimental study based on nuclear magnetic resonance and micro-CT," Energy and Fuels, vol. 34, no. 9, pp. 10894-10903, 2020.

[21] Y. L. Jiang, W. G. Liang, and Z. G. Li, "Experimental study on fracturing across coal-rock interfaces and the acoustic emission response characteristics," Chinese Journal of Rock Mechanics and Engineering, vol. 38, no. 5, pp. 875-887, 2018.

[22] Q. Meng, M. Zhang, L. Han, H. Pu, and T. Nie, "Effects of acoustic emission and energy evolution of rock specimens under the uniaxial cyclic loading and unloading compression," Rock Mechanics and Rock Engineering, vol. 49, no. 10, pp. 3873-3886, 2016.

[23] A. D. Shabdirova, F. Khamitov, and A. A. Kozhagulova, "Experimental and numerical investigation of the plastic zone permeability," in Proceedings of the 54th US Rock Mechanics/ Geomechanics Symposium, American Rock Mechanics Association, Colarado, CA, USA, July 2020.

[24] A. B. Zhuravlev, V. I. Karev, Y. F. Kovalenko, and K. B. Ustinov, "The effect of seepage on the stress-strain state of rock near a borehole," Journal of Applied Mathematics and Mechanics, vol. 78, no. 1, pp. 56-64, 2014.

[25] M. Ortiz and A. Pandolfi, "Finite-deformation irreversible cohesive elements for three-dimensional crack-propagation analysis," International Journal for Numerical Methods in Engineering, vol. 44, no. 9, pp. 1267-1282, 1999.

[26] K. Park, G. H. Paulino, and J. R. Roesler, "Virtual internal pair-bond model for quasi-brittle materials," Journal of Engineering Mechanics, vol. 134, no. 10, pp. 856-866, 2008. 
[27] X. Men, S. Tao, Z. Liu, W. Tian, and S. Chen, "Experimental study on gas mass transfer process in a heterogeneous coal reservoir," Fuel Processing Technology, vol. 216, Article ID 106779, 2021

[28] J. F. Yang, "Study on the constitutive equation of the cohesive crack in coals and its application in fracturing engineering," Dissertation, Taiyuan University of Technology, Taiyuan, China, 2019.

[29] H. Li, "Study on the rock characteristics of plasticity and fluidsolid coupling under confining pressure and its application," Dissertation, China University of mining and technology, China, 2018.

[30] Y. B. Zhu, "Pore structure characteristic and transport property analysis of porous medium," Dissertation, Henan University of Technology, Henan, China, 2015.

[31] Q. Zhang, C. G. Ge, and W. Li, "A new model and application of coalbed methane high efficiency production from broken soft and low permeable coal seam by roof strata-in horizontal well and staged hydraulic fracture," Journal of China Coal Society, vol. 43, no. 1, pp. 150-159, 2018.

[32] Y. Hao, P. Fu, and C. R. Carrigan, "Application of a dualcontinuum model for simulation of fluid flow and heat transfer in fractured geothermal reservoirs," in Proceedings of the 38th Stanford Geothermal Workshop, California, CA, USA, February 2013.

[33] A. Sakhaee-Pour and M. F. Wheeler, "Effective flow properties for cells containing fractures of arbitrary geometry," $S P E$ Journal, vol. 21, no. 03, pp. 0965-0980, 2016. 